\title{
Guest Editorial \\ Foreword to the special Issue of the XVII Symposium on Virtual and Augmented Reality - SVR 2015
}

This special issue of the JIS (SBC Journal on Interactive Systems) acknowledges the best papers of the XVII Symposium on Virtual and Augmented Reality (SVR 2015). SVR - the most important event on Virtual and Augmented Reality in Brazil in the last 17 years. Academic and professional members of the Brazilian Computer Society (SBC) support the conference since its beginning.

To attend reader expectations, the selected papers come from different sub-areas of the Virtual and Augmented Reality. The developments introduced in the papers reflect important recent advances and achievements by the community. More specifically, this issue includes studies on crowd simulation, gesture driven interaction, the use of a camera as a pointing device, and tracking by applying grayscale conversion.

In the first paper "A panoramic synthetic vision model for improving collision avoidance in crowd Simulation", the authors propose an improvement on models for crowd simulation in which agents are equipped with a synthetic vision system, by upgrading the agent's visual system allowing its vision beyond $180 \mathrm{o}$. This paper also analyzes the parameters used to define the field of view and their influence on the agent's behavior and on the efficiency of the algorithms.

The second paper, entitled "Gesture-Driven Interaction Using the Leap Motion to Conduct a 3D Particle System: Evaluation and Analysis of an Orchestral Performance" evolves the use of gestural interfaces by using a Leap Motion Controller. The authors present and evaluate an interactive simulation of $3 \mathrm{D}$ particles conducted by the Leap Motion, for an orchestral arrangement. As an application scenario, the proposed system facilitates the understanding of musical compositions for an ensemble of instruments, while emphasizing the importance of the conductor for a performance of a musical piece. This is achieved through the visual mapping of abstract sound elements (timbre or tone color, volume or sound intensity, and pitch), conducting patterns and music notation scores.

The authors of the third paper " $5 *$ Magic Wand: a RGBD camera-based 5 DoF pointing device for $3 D$ interaction" propose an expensive $3 \mathrm{D}$ pointing device. The tracking of the wand, which is an inexpensive stick, is based on computer vision and it was tested using a standard $\mathrm{PC}$ and RGBD camera.

Finally, the paper "A comparative study of grayscale conversion techniques applied to descriptor based tracking" examines the impact of color to grayscale conversion techniques on tracking and homography calculation results, which are fundamental to augmented reality applications.

We would like to thank the authors and all reviewers for offering wonderful feedback and advice.

We would also like to express our gratitude to Alberto Raposo for giving us the opportunity to organize this special issue.

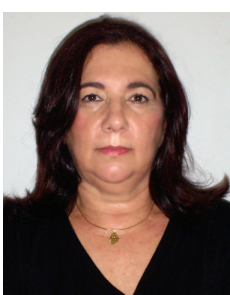

Judith Kelner received a First Degree in Civil Engineering (76) at the Federal University of Pernambuco (UFPE), Brazil and a Ph.D. at University of Kent at Canterbury, England (93). Actually, she is a Full Professor at Computer Science Center of UFPE. Her research interests include computer vision and networks areas acting on the following topics: augmented reality, tracking and 3D reconstruction, advanced interfaces, Internet of things and cloud computing.

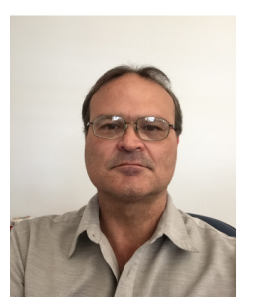

Eduardo Albuquerque received a First Degree in Civil Engineering (85) at the University of Brasília (UNB), Brazil, a MsC at Federal University of Pernambuco (UFPE), Brazil (89) and a Ph.D. at University of Kent at Canterbury, England (95). He joined University Federal de Goiás (UFG), Brazil, in 95 as a lecturer and currently he is the head of the Department of Informatics at UFG. His research interests include multimedia authoring and applications, games, virtual reality and augmented reality. 